\title{
CUL4B ubiquitin ligase in mouse development: A model for human X-linked mental retardation syndrome?
}

\author{
Yongchao Zhao ${ }^{1}$, Yi Sun ${ }^{1}$
}

${ }^{1}$ Division of Radiation and Cancer Biology, Department of Radiation Oncology, University of Michigan, 4424B MS-1, 1301 Catherine Street, Ann Arbor, MI 48109, USA

Cell Research (2012) 22:1224-1226. doi:10.1038/cr.2012.79; published online 15 May 2012

CUL4B, a member of the cullinRING ubiquitin ligase family, is frequently mutated in $\mathrm{X}$-linked mental retardation (XLMR) patients. The study by Liu et al. showed that Cul4b plays an essential developmental role in the extra-embryonic tissues, while it is dispensable in the embryo proper during mouse embryogenesis. Viable Cul4b-null mice provide the first animal model to study neuronal and behavioral deficiencies seen in human CUL4B XLMR patients.

CUL4 is a member of the cullinRING ubiquitin ligase family, the largest E3 ligase family, which appears to account for $\sim 20 \%$ of total protein degradation by the ubiquitin-proteasome system [1-3]. CUL4 is conserved during evolution from yeast to human. In yeast, CUL4 encodes a single gene, but mammalian cells express two closely related paralogs, CUL4A and CUL4B with about $82 \%$ sequence identity. CUL4A and CUL4B assemble structurally similar E3 complexes through binding to an adaptor protein (DDB1) and a substrate receptor protein (DCAF) at the $\mathrm{N}$-terminus, and a RING protein RBX1 at the C-terminus (Figure 1), and share functional redundancy in targeting substrates such as p21 and Cdt1 for ubiquitination

Correspondence: Yi Sun

Tel: 734-615-1989; Fax: 734-763-1581

E-mail: sunyi@umich.edu and degradation $[1,2]$. The Cul4a-null mice are viable and display no abnormal development and growth phenotypes, likely due to functional compensation from Cul4b $[4,5]$. The only phenotype associated with $\mathrm{Cul} 4 \mathrm{a}$ abrogation is the reproductive defects seen with male but not female mice, resulting from differential non-overlapping expression patterns of the two Cul4 genes during male meiosis [6]. On the other hand, germline deletion of $C u l 4 b$ resulted in embryonic lethality around E9.5 [7], indicating a unique function of $\mathrm{Cul} 4 \mathrm{~b}$ that cannot be compensated by $\mathrm{Cul} 4 \mathrm{a}$ during embryogenesis.

Mental retardation (MR) affects approximately $1 \%-3 \%$ of the population and is about $30 \%$ more common in males than in females [8], suggesting a causal relationship with gene mutations on the $\mathrm{X}$ chromosome. To date, mutations in about 100 genes have been identified in X-linked MR (XLMR), much more than those found on autosomes [9]. In 2007, two independent groups reported that mutations of $C U L 4 B$ (Xq24) ubiquitin ligase gene are associated with XLMR $[10,11]$. CUL4B-deficient patients display a syndrome of delayed puberty, moderate short stature, hypogonadism, relative macrocephaly, central obesity, fine intention tremor, brachydactyly, and large tongue $[10,11]$. Similarly, the neuronal and developmental deficiencies found in XLMR patients with CUL4B mutations are not compensated by CUL4A. The studies of the molecular pathogenesis of human XLMR are lagging partly due to the lack of an animal model for the disease.

In the most recent study published in Cell Research, Zhou and coworkers [12] attempted to generate conditional $C u l 4 b$ knockout mice with targeted deletion of $C u l 4 b$ at exons 4 and 5, giving rise to a non-functional $\mathrm{Cul} 4 \mathrm{~b}$ fragment lacking both the DDB1-binding domain and the cullin homology domain for RBX1 recruitment. The chicken-actin (CAG)-Cre was used, which drives Cre-mediated recombination at the early zygote stage, leading to $\mathrm{Cul} 4 \mathrm{~b}$ deletion in both the embryo proper and extraembryonic tissues. Like human CUL4B, the mouse Cul4b is also located on the $\mathrm{X}$-chromosome. Intercrossing of male CAG-Cre with female Cul4b $b^{f /+}$ revealed that hemizygous deletion of $C u l 4 b$ causes embryonic lethality. No embryos with the genotype of $\mathrm{Cul}_{4} b^{-1 y}$ survived beyond E9.5. Interestingly, the heterozygous $\mathrm{Cul}_{4} \mathrm{~b}^{+/}$embryos also die in the uterus before E13.5, suggesting that the paternal $\mathrm{X}$ chromosome undergoes imprinted inactivation with only trace amount, if any, of Cul $4 b$ expression remaining in extra-embryonic tissues. Detailed analysis of dissected embryos revealed that dying $\mathrm{Cul}_{4} \mathrm{~b}^{+/}$ embryos (E12.5) lack blood supply from the yolk sacs, whereas the $\mathrm{Cul}_{4 b^{-y}}$ embryos (E8.5) showed remarkable reduction in proliferation with growth 


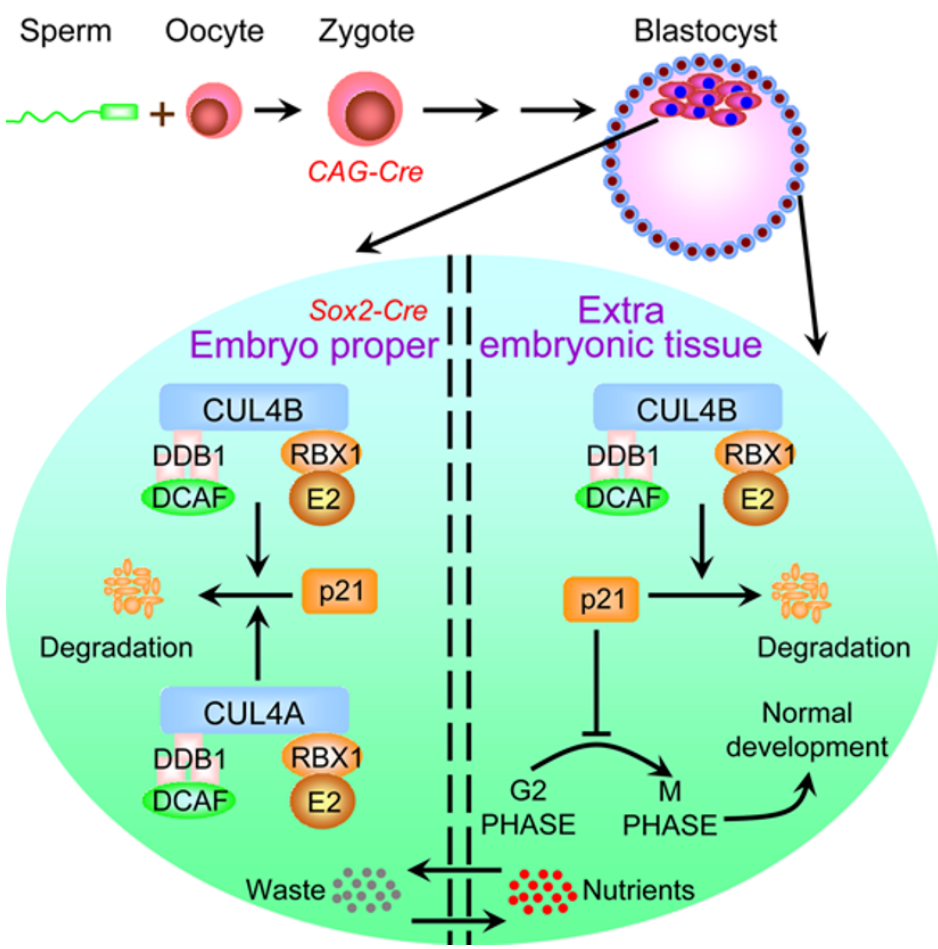

Figure 1 Differential expression of Cul4a and Cul4b in the embryo proper and extra embryonic tissues determines their fate. Before implantation, both Cul4a and $\mathrm{Cul} 4 \mathrm{~b}$ are expressed in the blastocyst. Following implantation, Cul4a is expressed in the embryo proper, but not in extra-embryonic tissues. Upon Cul4b deletion, p21 accumulates in extra-embryonic tissues to induce G2/M arrest and eventually embryonic death due to degeneration of extra-embryonic tissues. Expression of Cul4a in embryo prevents p21 accumulation and subsequent embryonic death.

arrest at G2/M and enhanced apoptosis. The authors went on and investigated why $\mathrm{Cul} 4 \mathrm{a}$ failed to compensate the loss of $C u l 4 b$, and found a dynamic expression pattern, differing between two forms, during early embryonic development. Prior to implantation, both Cul4 proteins are detectable in the blastocysts. Shortly after implantation, while both forms are expressed in the embryo proper, only Cul $4 \mathrm{~b}$ is expressed in the extra-embryonic tissues. Thus, upon $C u l 4 b$ deletion, extra-embryonic tissues without Cul4a compensation degenerate, eventually leading to embryonic death. Consistently, when the authors deleted $C u l 4 b$ in the epiblast using the Sox2-Cre (targeted Cul4b deletion in embryos proper only), viable $C u l 4 b$-null mice are produced likely due to Cul4a compensation. Thus, $C u l 4 b$ is essential for the development of extraembryonic tissues, but is dispensable for embryogenesis itself.

To study the potential underlying mechanism(s) of embryonic lethality upon $C u l 4 b$ deletion in extra-embryonic tissues, the authors used an extra-embryonic cell line (XEN). Cul4b knockdown induced a remarkable cell cycle arrest at the G2/M phase, consistent with observation made in Cul4b-null embryos, and robust accumulation of p21, a universal inhibitor of cyclin dependent kinase and a known substrate of Cul4 [1]. To determine whether accumulated p21 is responsible for the G2/M arrest, the authors simultaneously knocked down both Cul4b and $p 21$ in XEN cells and observed a partial abrogation of growth arrest, suggesting that p21 plays a causal role, at least in part.
Unfortunately, due to unavailability of anti-mouse p21 antibody specific for immunohistochemical staining, the authors were not able to show if $\mathrm{p} 21$ is indeed accumulated in extra-embryonic tissues upon $C u l 4 b$ deletion. However, whether $\mathrm{p} 21$ indeed plays a causal role in embryonic death upon $\mathrm{Cul} 4 \mathrm{~b}$ deletion can be unequivocally determined by a rescuing experiment in which simultaneous deletion of $p 21$ should abrogate or at least delay embryonic lethality, if it is causal. Nevertheless, the study by Zhou's group can be summarized as follows. Before implantation, both Cul4a and Cul4b ubiquitin ligases are expressed in the blastocyst (inner cell mass and trophoblast cells). Following embryo implantation, while Cul4b is expressed in both the embryo proper and extra embryonic tissues, Cul4a is only expressed in the embryo proper. The CAG-Cre-driven Cul $4 b$ deletion (in both the embryo proper and extra-embryonic tissues) causes significant $\mathrm{p} 21$ accumulation in Cul4a non-expressing extra-embryonic tissues, resulting in $\mathrm{G} 2 / \mathrm{M}$ arrest, followed by embryonic death due to degeneration of extraembryonic tissues. On the embryo side, $C u l 4 b$ deletion has no detrimental consequence, benefiting from the compensatory effect of Cul4a for p21 targeting. The same holds true when $C u l 4 b$ is deleted driven by embryonic specific Sox2-Cre (Figure 1).

It is noteworthy that the studies by Zhou's group revealed two distinct differences between $C u l 4 b \mathrm{KO}$ mice and CUL4B-associated XLMR patients. First, $C u l 4 b$ deletion at the zygote stage causes embryonic lethality, whereas XLMR patients with CUL4B mutations live to adulthood. Second, the Cul4bnull allele cannot be transmitted from the mother to the offspring, whereas human XLMR patients inherit X-linked CUL4B mutations from their mothers. Nevertheless, viable $C u l 4 b$-null mice (upon epiblast ablation by Sox2-Cre) provide the first mouse model for mechanistic study of human XLMR diseases associated with CUL4B mutations in the 
following three aspects:

First, as noted earlier, human CUL4B XLMR patients have multiple neuronal and developmental defects. An obvious follow-up study will be to use this mouse model for neurological and behavioral analyses to determine whether Cul4b-null mice indeed present some of human XLMR symptoms.

Second, this model can also be used to validate whether accumulation of $C u l 4 b$ substrates during various stages of brain development indeed plays a pathogenic role and contributes to the clinical symptoms of XLMR patients. For instance, WDR5, a recently identified gene affecting general cognitive ability [13], was found to be a novel nuclear substrate of CUL4B, but not CUL4A [14]. Investigation into whether WDR5 is abnormally accumulated upon Cul4b deletion in vivo would rule in or rule out its potential association with human XLMR, although it was not the case in this study using an extraembryonic cell line in vitro.

Third, the viability of $C u l 4 b$-null mice upon epiblast-specific deletion provides opportunities to study neuronal specific ablation of $C u l 4 b$ in association with the pathogenesis of CUL4B-associated XLMR. For example, Cul4b is expressed at high levels in the hippocampus and cerebrum of mouse brains; both regions are affected in MR patients [15]. Thus, the use of Cre mouse lines that target the deletion of $C u l 4 b$ in the entire brain, selected brain areas, or specific neuronal cells in both spatial and temporal manners [16] would reveal potential contributions of particular regions and cell types to the development and symptoms of CUL4Bassociated XLMR.

A number of questions that warrant future investigation remain unanswered. First, in addition to p21, what are the other Cul4B substrates, which also contribute to degeneration of extra-embryonic tissues upon $C u l 4 b$ deletion, since simultaneous deletion of p21 only partially rescues the growth defects? Second, besides the difference in tissue/cell specific expression seen in this study, are Cul4a and Cul4b targeting a unique set of substrates non-redundantly, thus differentiating their physiological functions? A related question will be why CUL4A cannot compensate for the loss of CUL4B in CUL4B-associated XLMR patients? Third, what is the pathogenic mechanism for CUL4B-associated XLMR? Is it mainly due to pathological accumulation of many CUL4B substrates? Answers to these questions may offer insights into potential therapeutic strategies for the treatment of CUL4Bassociated XLMR patients.

In summary, the findings reported by Zhou's group provide the first convincing evidence that demonstrates an essential role of $\mathrm{Cul} 4 \mathrm{~b}$ in the development of extra-embryonic tissues during mouse embryogenesis. The viable $\mathrm{Cul} 4 \mathrm{~b}$ conditional knockout mice, generated in this study, may serve as the first mouse model for future mechanistic studies of neuronal and behavioral deficiencies of human XLMR associated with CUL4B mutations. We look forward to more exciting discoveries of how $\mathrm{Cul} 4 \mathrm{~b}$ deficiency leads to the development of XLMR in years to come.

\section{References}

1 Jackson S, Xiong Y. CRL4s: the CUL4RING E3 ubiquitin ligases. Trends Biochem Sci 2009; 34:562-570.

2 Lee J, Zhou P. DCAFs, the missing link of the CUL4-DDB1 ubiquitin ligase. Mol Cell 2007; 26:775-780.

3 Soucy TA, Smith PG, Milhollen MA, et al. An inhibitor of NEDD8-activating enzyme as a new approach to treat cancer. Nature 2009; 458:732-736.

4 Liu L, Lee S, Zhang J, et al. CUL4A abrogation augments DNA damage response and protection against skin carcinogenesis. Mol Cell 2009; 34:451-460.

5 Yin Y, Lin C, Kim ST, et al. The E3 ubiquitin ligase Cullin 4A regulates meiotic progression in mouse spermatogenesis. Dev Biol 2011; 356:51-62.

6 Kopanja D, Roy N, Stoyanova T, Hess RA, Bagchi S, Raychaudhuri P. Cul4A is essential for spermatogenesis and male fertility. Dev Biol 2011; 352:278-287.

7 Cox BJ, Vollmer M, Tamplin O, et al. Phenotypic annotation of the mouse X chromosome. Genome Res 2010; 20:1154-1164.

8 Larson SA, Lakin KC, Anderson L, Kwak N, Lee JH, Anderson D. Prevalence of mental retardation and developmental disabilities: estimates from the 1994/1995 National Health Interview Survey Disability Supplements. Am J Ment Retard 2001; 106:231-252.

9 Chiurazzi P, Schwartz CE, Gecz J, Neri G. XLMR genes: update 2007. Eur J Hum Genet 2008; 16:422-434.

10 Tarpey PS, Raymond FL, O'Meara S, et al. Mutations in CUL4B, which encodes a ubiquitin E3 ligase subunit, cause an $\mathrm{X}$-linked mental retardation syndrome associated with aggressive outbursts, seizures, relative macrocephaly, central obesity, hypogonadism, pes cavus, and tremor. Am J Hum Genet 2007; 80:345352.

11 Zou Y, Liu Q, Chen B, et al. Mutation in CUL4B, which encodes a member of cullin-RING ubiquitin ligase complex, causes X-linked mental retardation. Am J Hum Genet 2007; 80:561-566.

12 Liu L, Yin Y, Li Y, et al. Essential role of the CUL4B ubiquitin ligase in extraembryonic tissue development during mouse embryogenesis. Cell Res 2012; 22:1258-1269.

13 Davis OS, Butcher LM, Docherty SJ, et al. A three-stage genome-wide association study of general cognitive ability: hunting the small effects. Behav Genet 2010; 40:759-767.

14 Nakagawa T, Xiong Y. X-linked mental retardation gene CUL4B targets ubiquitylation of $\mathrm{H} 3 \mathrm{~K} 4$ methyltransferase component WDR5 and regulates neuronal gene expression. Mol Cell 2011; 43:381-391.

15 Lein ES, Hawrylycz MJ, Ao N, et al. Genome-wide atlas of gene expression in the adult mouse brain. Nature 2007; 445:168-176.

16 Gaveriaux-Ruff C, Kieffer BL. Conditional gene targeting in the mouse nervous system: Insights into brain function and diseases. Pharmacol Ther 2007; 113:619-634. 